PROCEEDINGS OF THE

AMERICAN MATHEMATICAL SOCIETY

Volume 128, Number 11, Pages 3399-3403

S 0002-9939(00)05421-6

Article electronically published on May 11, 2000

\title{
SUPERPOSITION OPERATOR IN SOBOLEV SPACES ON DOMAINS
}

\author{
DENIS A. LABUTIN
}

(Communicated by Christopher D. Sogge)

\begin{abstract}
For an arbitrary open set $\Omega \subset \mathbb{R}^{n}$ we characterize all functions $G$ on the real line such that $G \circ u \in W^{1, p}(\Omega)$ for all $u \in W^{1, p}(\Omega)$. New element in the proof is based on Maz'ya's capacitary criterion for the imbedding $W^{1, p}(\Omega) \hookrightarrow L^{\infty}(\Omega)$.
\end{abstract}

Let $\Omega$ be an open set in $\mathbb{R}^{n}, n \geq 2$, and let $W^{1, p}(\Omega)$ be a Sobolev space with the norm

$$
\|u\|_{W^{1, p}(\Omega)}=\|u\|_{L^{p}(\Omega)}+\|\nabla u\|_{L^{p}(\Omega)}, \quad 1 \leq p<\infty .
$$

Let $G: \mathbb{R}^{1} \rightarrow \mathbb{R}^{1}$ be a Borel function. Then the associated superposition operator $T_{G}$ is given by

$$
u \mapsto G \circ u .
$$

The purpose of the present paper is to find the necessary and sufficient conditions on $G$ for

$$
T_{G}: W^{1, p}(\Omega) \rightarrow W^{1, p}(\Omega) .
$$

This and more general problems were considered for $p \neq n$ and domains $\Omega$ with Lipschitz boundaries in [9], 10], and for $\Omega=\mathbb{R}^{n}, 1 \leq p<\infty$, in 3]. Following [3] we say that space $W^{1, p}(\Omega)$ is supercritical if $W^{1, p}(\Omega) \hookrightarrow L^{\infty}(\Omega)$, and subcritical if $W^{1, p}(\Omega) \hookrightarrow L^{\infty}(\Omega)$. By $|E|$ we denote Lebesgue measure of the set $E \subset \mathbb{R}^{n}$. Let $B(x, r)$ denote an open ball in $\mathbb{R}^{n}$ with center $x$ and radius $r$.

The core of the works [10], [3] is the criterion for (1) when $\Omega$ is a cube. If $\Omega$ is a cube, then (1) is equivalent to $G^{\prime} \in L^{\infty}\left(\mathbb{R}^{1}\right)$ for subcritical $W^{1, p}(\Omega)$ (that is, for $1 \leq p \leq n$ ), and to $G^{\prime} \in L_{\text {loc }}^{\infty}\left(\mathbb{R}^{1}\right)$ for supercritical $W^{1, p}(\Omega)$ (that is, for $p>n$ ). As a direct consequence of this result we obtain that for an arbitrary open set $\Omega \subset \mathbb{R}^{n}$

$$
T_{G}: W_{\mathrm{loc}}^{1, p}(\Omega) \rightarrow W_{\mathrm{loc}}^{1, p}(\Omega)
$$

is equivalent to $G^{\prime} \in L^{\infty}\left(\mathbb{R}^{1}\right)$ if $1 \leq p \leq n$, and to $G^{\prime} \in L_{\text {loc }}^{\infty}\left(\mathbb{R}^{1}\right)$ if $p>n$. Moreover, if for a domain $\Omega,|\Omega|<\infty$, the exponent $p=n$, as in the Sobolev imbedding theorem, separates subcritical and supercritical $W^{1, p}(\Omega)$, then (1) is equivalent to (2). It seems that the broadest class of such domains $\Omega$ is the class

Received by the editors August 1, 1998 and, in revised form, January 22, 1999.

1991 Mathematics Subject Classification. Primary 46E35; Secondary 47H30.

Key words and phrases. Sobolev spaces, superposition operator.

This work was supported by the Russian Foundation for Basic Research grant 96-01-00243.

(C)2000 American Mathematical Society 
of John domains, 6], [2], 5]. For domains with infinite measure one has analogous results provided the condition $G(0)=0$ is added to the restrictions on $G^{\prime}$, [10], [3].

For an arbitrary $\Omega(1)$ is not equivalent to (2). For general open sets $\Omega$ our main result is Theorem 1 below. For an arbitrary $\Omega$ in subcritical case with $p>n$ the condition for (2) is necessary but not sufficient for (1). On the other hand, condition $G^{\prime} \in L^{\infty}\left(\mathbb{R}^{1}\right)$ is obviously sufficient for (1), but not necessary for (2) with $p>n$. In this case, in contrast with local constructions in [10, 3], we establish estimates near the boundary.

Our consideration is based on the following criterion for $W^{1, p}(\Omega) \hookrightarrow L^{\infty}(\Omega)$, which was proved by Maz'ya. For $x \in \Omega$ we define

$$
V_{\Omega}(x)=\left\{f \in C_{\mathrm{loc}}^{\infty}(\Omega): 0 \leq f \leq 1, f(x)=1\right\} .
$$

Relative $p$-capacity in $\Omega$ of a point $x \in \Omega$ with respect to the ball $B(x, r)$ is defined (see [7]) as

$$
\operatorname{cap}_{p}(x, B(x, r) ; \Omega)=\inf \left\{\int_{\Omega}|\nabla f|^{p}: f \in V_{\Omega}(x),\left.f\right|_{\Omega \backslash B(x, r)} \equiv 0\right\} .
$$

Maz'ya [7] proved that $W^{1, p}(\Omega) \hookrightarrow L^{\infty}(\Omega)$ if and only if

$$
\inf \left\{\operatorname{cap}_{p}(x, B(x, r) ; \Omega): x \in \Omega\right\}=0 \quad \text { for all } r>0 .
$$

Note that for $B(x, r) \subset \subset \Omega$ and $p>n$

$$
\operatorname{cap}_{p}(x, B(x, r) ; \Omega)=c(n, p) r^{n-p} \rightarrow \infty \quad \text { as } r \rightarrow 0
$$

(see 7]).

It should be mentioned that there is a vast literature concerning characterisation of $G$ for $T_{G}$ to act in Besov and Lizorkin-Triebel spaces on $\mathbb{R}^{n}$. We refer to surveys 4], 12, [13, and to the book 11. Results for superposition operators in other spaces of real functions, such as Lebesgue spaces, $B V$, and ideal spaces, can be found in [1].

Theorem 1. Let $\Omega \subset \mathbb{R}^{n}$ be an open set, $|\Omega|<\infty, 1 \leq p<\infty$. The following conditions on the function $G: \mathbb{R}^{1} \rightarrow \mathbb{R}^{1}$ are necessary and sufficient for $T_{G}$ : $W^{1, p}(\Omega) \rightarrow W^{1, p}(\Omega):$

(i) $G^{\prime} \in L^{\infty}\left(\mathbb{R}^{1}\right)$, if $W^{1, p}(\Omega)$ is subcritical.

(ii) $G^{\prime} \in L_{\mathrm{loc}}^{\infty}\left(\mathbb{R}^{1}\right)$, if $W^{1, p}(\Omega)$ is supercritical.

The proof of the theorem is given at the end of the paper. First we make some remarks.

Remark 2. Condition (3) is equivalent to $W^{1, p}(\Omega) \hookrightarrow L^{\infty}(\Omega)$ also in the case $|\Omega|=$ $\infty$. For open sets $\Omega$ of infinite measure Theorem 1 is valid provided we add the obvious necessary condition $G(0)=0$ in both (i) and (ii). The proof is the same as below.

Remark 3. Let $\Omega$ be a connected open set and let $\omega$ be an open nonempty set with compact closure $\bar{\omega} \subset \Omega$. Following [7] we introduce the Banach space $L^{1, p}(\Omega)$ with the norm

$$
\|u\|_{L^{1, p}(\Omega)}=\|u\|_{L^{p}(\omega)}+\|\nabla u\|_{L^{p}(\Omega)}
$$


It is known that norms corresponding to different choices of $\omega$ are equivalent (see [7]). If $|\Omega|<\infty$ then condition (3) is equivalent to $L^{1, p}(\Omega) \nrightarrow L^{\infty}(\Omega)$ (see [7]). Theorem 1 holds for the space $L^{1, p}(\Omega)$ whenever $|\Omega|<\infty$. The proof is even easier than for $W^{1, p}(\Omega)$, as we do not need to control $\|u\|_{L^{p}(\Omega)}$.

Remark 4. From [9] and [10] it follows that the nonlinear operator $T_{G}$ in Theorem 1 is bounded and continuous in $W^{1, p}(\Omega)$ as soon as (1) is valid. The same is true for $T_{G}$ in $L^{1, p}(\Omega)$ provided $|\Omega|<\infty$, and in $W^{1, p}(\Omega)$ if $|\Omega|=\infty$.

Proof of Theorem 1 . The only part of the theorem not covered by conditions from [3] for (2) is the necessity for $p>n$ in (i).

Thus we need to prove that if $T_{G}: W^{1, p}(\Omega) \rightarrow W^{1, p}(\Omega), p>n$, and $W^{1, p}(\Omega) \hookrightarrow$ $L^{\infty}(\Omega)$, then $G^{\prime} \in L^{\infty}\left(\mathbb{R}^{1}\right)$. From [9] and [10] it follows that $G$ is absolutely continuous on $\mathbb{R}^{1}$ and $G^{\prime} \in L_{\text {loc }}^{\infty}\left(\mathbb{R}^{1}\right)$. Moreover, for all $u \in W^{1, p}(\Omega)$ one has $\nabla(G \circ u)=G^{\prime}(u) \nabla u$ a.e. in $\Omega$.

Seeking a contradiction suppose that $G^{\prime} \notin L^{\infty}\left(\mathbb{R}^{1}\right)$. Then we shall construct a function $U \in W^{1, p}(\Omega)$ such that $\|\nabla(G \circ U)\|_{L^{p}(\Omega)}=\infty$. To do this we first construct a special sequence of functions $\left\{u_{j}\right\}, u_{j} \in W^{1, p}(\Omega) \cap L^{\infty}(\Omega) \cap C_{\mathrm{loc}}(\Omega)$ for $j=1$, $2, \ldots$, and a sequence of pairwise disjoint balls $\left\{B\left(y_{j}, \rho_{j}\right)\right\}=\left\{B_{j}\right\}, B_{j} \subset \subset \Omega$, with the properties

$$
\begin{gathered}
\left\|u_{j}\right\|_{L^{p}(\Omega)}+\left(\int_{\Omega \backslash \bigcup_{m \leq j} B_{m}}\left|\nabla u_{j}\right|^{p}\right)^{1 / p} \leq\left(1+\cdots+\frac{1}{2^{j}}\right) \text { for all } j \geq 1, \\
\left.u_{m}\right|_{B_{j}}=\left.u_{j}\right|_{B_{j}} \text { for } m \geq j .
\end{gathered}
$$

The following inequalities are valid for all $j \geq 1$ :

$$
\begin{gathered}
\int_{B_{j}}\left|\nabla u_{j}\right|^{p} \leq 1 / j^{2}, \\
\int_{B_{j}}\left|G^{\prime}\left(u_{j}\right)\right|^{p}\left|\nabla u_{j}\right|^{p} \geq C(n),
\end{gathered}
$$

with some $C(n)>0$ independent of $j$.

By (5) - (17) the sequence $\left\|u_{j}\right\|_{W^{1, p}(\Omega)}$ is bounded, and we can extract a subsequence converging weakly to some function $U \in W^{1, p}(\Omega)$. Now (6) and (8) imply that $\nabla(G \circ U) \notin L^{p}(\Omega)$.

We construct the sequences $\left\{u_{j}\right\}$ and $\left\{B_{j}\right\}$ by induction. Using (4) we choose a constant $R=R(n, p)$ such that for $B(z, R) \subset \subset \Omega$

$$
\operatorname{cap}_{p}(z, B(z, R) ; \Omega) \geq 2 .
$$

Without loss of generality we can assume that $G^{\prime} \notin L^{\infty}\left(\mathbb{R}_{+}^{1}\right)$, where $\mathbb{R}_{+}^{1}=(0,+\infty)$.

To construct $u_{1}$ we take the number $t_{1}$ such that $t_{1}>1,\left|G^{\prime}\left(t_{1}\right)\right| \geq 1$ and $t_{1}$ is a Lebesgue point of $G^{\prime}$. The latter implies that

$$
\left|\left\{s:\left|t_{1}-s\right| \leq \delta,\left|G^{\prime}(s)\right| \geq 1\right\}\right| \geq \delta
$$

for all sufficiently small $\delta$. Next we choose $0<r_{1}<R$ so small that $t_{1}\left|B\left(0, r_{1}\right)\right|^{1 / p}<$ $1 / 4$. For these $t_{1}$ and $r_{1}$ using (3) we find a ball $B\left(y_{1}, r_{1}\right), y_{1} \in \Omega$, and a function $v_{1} \in V_{\Omega}\left(y_{1}\right)$ supported in $B\left(y_{1}, r_{1}\right) \cap \Omega$ such that $\int_{\Omega}\left|\nabla v_{1}\right|^{p} \leq 1 /\left(4 t_{1}\right)^{p}$, or 
equivalently

$$
\left(\int_{\Omega}\left|\nabla\left(2 t_{1} v_{1}\right)\right|^{p}\right)^{1 / p} \leq 1 / 2 .
$$

Now we put $w_{1}=\min \left\{t_{1}, 2 t_{1} v_{1}\right\}$. Note that truncating does not increase norm in $W^{1, p}(\Omega)$. Thus because of our choice of $r_{1}$ we have

$$
\left\|w_{1}\right\|_{W^{1, p}(\Omega)} \leq\left\|2 t_{1} v_{1}\right\|_{W^{1, p}(\Omega)} \leq 1 .
$$

Note that $v_{1}\left(y_{1}\right)=1$. Consequently there exists a ball $B_{1}=B\left(y_{1}, \rho_{1}\right) \subset \subset \Omega$ such that $\left.w_{1}\right|_{B_{1}} \equiv t_{1}$. To finish construction of $u_{1}$ we use the function $\phi(x)=$ $\max \{1-|x|, 0\}, x \in \mathbb{R}^{n}$. We denote $\phi_{\varepsilon}(x)=\phi(x / \varepsilon)$ for $\varepsilon>0$. We can choose $a>0$ so small that (10) is satisfied for $\delta=a$, and $\varepsilon<\rho_{1}$ such that

$$
\begin{gathered}
\left\|a \phi_{\epsilon}\right\|_{L^{p}\left(\mathbb{R}^{n}\right)} \leq C_{1}(n) a \varepsilon^{n / p} \leq 1 / 2, \\
\int_{\mathbb{R}^{n}}\left|\nabla\left(a \phi_{\varepsilon}\right)\right|^{p}=C_{2}(n) a^{p} / \varepsilon^{p-n}=1 / 2 .
\end{gathered}
$$

Note that (10) and the linear radial nature of $\phi$ imply that for such $a$

$$
\left|\left\{z \in \mathbb{R}^{n}:|z|<\varepsilon,\left|G^{\prime} \circ\left(t_{1}+a \phi_{\varepsilon}(z)\right)\right| \geq 1\right\}\right| \geq C_{3}(n) \varepsilon^{n} .
$$

Therefore one also has

$$
\int_{\mathbb{R}^{n}}\left|\nabla\left(G \circ\left(t_{1}+a \phi_{\varepsilon}\right)\right)\right|^{p} \geq C_{3}(n) a^{p} / \varepsilon^{p-n} \geq C_{3}(n) / 2 C_{2}(n) .
$$

Now taking $u_{1}(x)=w_{1}(x)+a \phi_{\varepsilon}\left(x-y_{1}\right), x \in \Omega$, we see that $u_{1} \in W^{1, p}(\Omega) \cap$ $L^{\infty}(\Omega) \cap C_{\mathrm{loc}}(\Omega)$ and (5), (7), (8) are valid for $j=1$.

Now we construct $u_{k}$ assuming that the functions $u_{1}, \ldots, u_{k-1}$ and the balls $B_{1}, \ldots, B_{k-1}$ with all required properties have already been constructed. We can find $t_{k}$ such that

$$
t_{k} \geq 4\left\|u_{k-1}\right\|_{L^{\infty}(\Omega)}
$$

$\left|G^{\prime}\left(t_{k}\right)\right| \geq k$, and $t_{k}$ is a Lebesgue point of $G^{\prime}$. Next we choose $0<r_{k}<R$ so small that

$$
4 r_{k} \leq \min \left\{\operatorname{dist}\left(B_{j}, \partial \Omega\right), j=1, \ldots, k-1\right\},
$$

and $t_{k}\left|B\left(0, r_{k}\right)\right|^{1 / p}<1 / 2^{k+2}$. For $r_{k}, t_{k}$ we find, using (3), a ball $B\left(y_{k}, r_{k}\right), y_{k} \in \Omega$, and a function $v_{k} \in V_{\Omega}\left(y_{k}\right)$ supported in $B\left(y_{k}, r_{k}\right) \cap \Omega$ such that

$$
\left(\int_{\Omega}\left|\nabla\left(2 t_{k} v_{k}\right)\right|^{p}\right)^{1 / p} \leq 1 / 2^{k+2} .
$$

We note that (9) and (14) imply that $\partial B\left(y_{k}, r_{k}\right) \cap \partial \Omega \neq \emptyset$. Thus $B\left(y_{k}, r_{k}\right)$ does not intersect any of $B_{1}, \ldots, B_{k-1}$ because of (13). We define $w_{k}$ in a similar way as $w_{1}$. Let $w_{k}=\min \left\{t_{k}, u_{k-1}+2 t_{k} v_{k}\right\}$. Because of (12) $w_{k}$ coincides with $u_{k-1}$ in the balls $B_{1}, \ldots, B_{k-1}$. From (12) one has $u_{k-1}\left(y_{k}\right)+2 t_{k} v_{k}\left(y_{k}\right)>t_{k}$. Therefore there is a ball $B_{k}=B\left(y_{k}, \rho_{k}\right) \subset \subset \Omega, B_{k} \subset B\left(y_{k}, r_{k}\right)$, not intersecting $B_{1}, \ldots$, $B_{k-1}$, such that $\left.w_{k}\right|_{B_{k}} \equiv t_{k}$. By induction we have the estimate

$$
\left\|w_{k}\right\|_{W^{1, p}(\Omega)} \leq\left(1+\ldots+\frac{1}{2^{k-1}}+\frac{1}{2^{k+1}}\right) .
$$


To construct $u_{k}$ we modify $w_{k}$ in the ball $B_{k}$. We use the function $a \phi_{\varepsilon}$ defined as above. We choose $a$ and $\varepsilon$ such that $\operatorname{supp}\left(a \phi_{\varepsilon}\right) \subset B_{k}$,

$$
\begin{gathered}
\left|\left\{s:\left|t_{k}-s\right| \leq a,\left|G^{\prime}(s)\right| \geq k\right\}\right| \geq a, \\
\left\|a \phi_{\varepsilon}\right\|_{L^{p}\left(\mathbb{R}^{n}\right)} \leq 1 / 2^{k+1}, \\
\int_{\mathbb{R}^{n}}\left|\nabla\left(a \phi_{\varepsilon}\right)\right|^{p}=1 / k^{2} .
\end{gathered}
$$

By analogy with (11) from (15) and (16) we have

$$
\int_{\mathbb{R}^{n}}\left|\nabla\left(G \circ\left(t_{k}+a \phi_{\varepsilon}\right)\right)\right|^{p} \geq C_{4}(n) k^{p} / k^{2} \geq C_{5}(n) .
$$

Now we define $u_{k}(x)=w_{k}(x)+a \phi_{\varepsilon}\left(x-y_{k}\right)$, and (5) -(8) hold. This completes the proof.

\section{REFERENCES}

1. J. Appel, P. P. Zabrejko, Nonlinear superposition operators, Cambridge Tracts in Mathematics, 95, Cambridge University Press, Cambridge, 1990. MR 91k:47168

2. B. Bojarski, Remarks on Sobolev imbedding inequalities, Complex analysis, Joensuu 1987, 52-68, Lecture Notes in Math., 1351, Springer, Berlin-New York, 1988. MR 90b:46068

3. G. Bourdaud, Le calcul fonctionnel dans les espaces de Sobolev, Invent. Math. 104 (1991), 435-446. MR 93b:46053

4. G. Bourdaud, The functional calculus in Sobolev spaces, Function spaces, differential operators and nonlinear analysis (Friedrichroda, 1992), 127-142, Teubner-Texte Math., 133, Teubner, Stuttgart, 1993. MR 94e:46055

5. S. Buckley, P. Koskela, Sobolev-Poincare implies John, Math. Res. Lett. 2 (1995), no. 5, 577-593. MR 96i:46035

6. V. M. Gol'dshtein, Yu. G. Reshetnyak, Quasiconformal mappings and Sobolev spaces, Kluwer Academic Publishers Group, Dordrecht, 1990. MR 92h:46040

7. V. G. Maz'ya, Sobolev spaces, Springer-Verlag, Berlin-New York, 1985. MR 87g:46056

8. M. Marcus, V. J. Mizel, Absolute continuity on tracks and mappings of Sobolev spaces, Arch. Rational Mech. Anal. 45 (1972), 294-320. MR 49:3529

9. M. Marcus, V. J. Mizel, Every superposition operator mapping one Sobolev space into another is continuous, J. Funct. Anal. 33 (1979), 217-229. MR 80h:47039

10. M. Marcus, V. J. Mizel, Complete characterization of functions which act, via superposition, on Sobolev spaces, Trans. Amer. Math. Soc. 251 (1979), 187-218. MR 80j:46055

11. T. Runst, W. Sickel, Sobolev spaces of fractional order, Nemytskij operators, and nonlinear partial differential equations, de Gruyter Series in Nonlinear Analysis and Applications, 3. Walter de Gruyter Co., Berlin, 1996. MR 98a:47071

12. W. Sickel, Superposition of functions in Sobolev spaces of fractional order. A survey, Partial differential equations, 481-497, Banach Center Publ., 27, Part 1, 2, Polish Acad. Sci., Warsaw, 1992. MR 94e:46065

13. W. Sickel, Composition operators acting on Sobolev spaces of fractional order-a survey on sufficient and necessary conditions, Function spaces, differential operators and nonlinear analysis (Paseky nad Jizerou, 1995), 159-182, Prometheus, Prague, 1996. CMP 98:04

Centre for Mathematics and its Applications, School of Mathematical Sciences, Australian National University, Canberra 0200, ACT, Australia

E-mail address: labutin@maths.anu.edu.au 